\title{
Confirmation of No Influence of Loa loa and Mansonella perstans on the Card Agglutination Test for Trypanosomosis used for Serological Screening of Human African Trypanosomosis
}

Pion SD ${ }^{1}$, Njiokou $F^{2}$, Boussinesq $\mathbf{M}^{1}$, Simo $\mathbf{G}^{3}$ and Truc $\mathrm{P}^{4^{*}}$

${ }^{1}$ French National Research Institute for Sustainable Development IRD, Centre of Montpellier, Montpellier, France

${ }^{2}$ Laboratory of Parasitology and Ecology, Department of Biology and Animal Physiology, Faculty of Science, University of Yaoundé, Cameroon

${ }^{3}$ Unit of Parasitology and Molecular Entomology, Department of Biochemistry, Faculty of Science, University of Dschang, Dschang, Cameroon

${ }^{4}$ French National Research Institute for Sustainable Development IRD, Campus International de Baillarguet, Montpellier, France

*Corresponding author: Philippe Truc, French National Research Institute for Sustainable Development IRD UMR 177 INTERTRYP IRD CIRAD, Laboratory for Research and Coordination on Trypanosomoses, TA A-17/G, Baillarguet International Campus 34398 Montpellier Cedex 5, France, Tel: +33-611888828; Fax: +33467593894; E-mail: philippe.truc@ird.fr

Received date: July 26, 2017; Accepted date: August 04, 2017; Published date: August 16, 2017

Copyright: (C) 2017 Pion SD, et al. This is an open-access article distributed under the terms of the Creative Commons Attribution License, which permits unrestricted use, distribution, and reproduction in any medium, provided the original author and source are credited.

\begin{abstract}
In central Africa, the geographical distribution of human African trypanosomosis (HAT) due to Trypanosoma brucei gambiense overlaps that of Loa loa and Mansonella perstans filariases. This study investigated whether the presence of blood borne M. perstans and L. loa microfilariae interferes with the agglutination reaction of CATT (Card Agglutination Test for Trypanosomosis), used for mass screening of HAT. 146 CATT positive participants and 146 age and sex matched CATT negative subjects were recruited in three sites in Cameroon and one in the Republic of Congo. CATT positive persons were not more frequently infected by $L$. loa and $M$. perstans than CATT negative ones. This unique matched case-control study confirmed a previous study and does not bring any evidence of the influence of $L$. loa or $M$. perstans on serodiagnosis of HAT in the field using CATT/ T. b. gambiense LiTat 1.3. HAT screening activities can be performed without controlling for filariasis at the same time.
\end{abstract}

Keywords: Human African trypanosomosis; Microfilariasis; Loa loa; Mansonella perstans; CATT/ Trypanosoma brucei gambiense

\section{Introduction}

The card agglutination test for trypanosomosis (CATT/ Trypanosoma brucei gambiense) is a serological test that detects $T$. $b$. gambiense specific antibodies in whole blood, serum or plasma [1]. Easy to use in field conditions, this low-cost test remains the mainstay for mass population screening for the gambiense form of human African trypanosomosis (HAT) since 1978. In case of positive CATT, blood centrifugation and lumbar puncture to obtain cerebrospinal fluid are subsequently performed for confirmation and staging of the infection.

Despite a remarkable specificity, estimated around 97\% false positivity to the CATT may occur in case of a transient infection by an animal trypanosome such Trypanosoma brucei brucei or in individuals presenting with malaria [2]. It has also been suspected with filarial infections [3].

In 1988, the association between the presence of Loa loa and Mansonella perstans microfilaremia and CATT results was investigated in the Republic of Congo as part of a study whose main objective was to compare the performances of the CATT using the antigen consisted of clone trypanosomes of Variable Antigen Type (VAT) AnTat 1.8, isolated from Trypanosoma brucei brucei Antigen Repertoire AnTAR 1 [4], and the immunofluorescent antibody test (IFAT) as immunological diagnosis of HAT [3].
A total of 115 subjects infected with $L$. loa (defined by a history of Calabar swelling or of eyeworm, or the presence of blood microfilariae) and/or M. perstans (defined by the presence of blood microfilariae) and who were not exposed to the risk of HAT were tested with CATT using serum. Among these individuals, $29.6 \%$ were positive at the CATT, whereas none of the 105 individuals included in a "healthy patients" group (i.e., without HAT, filariasis, schistosomiasis or toxoplasmosis) were CATT positive. Prima facie, these results suggest that a filarial infection can induce a positive CATT result. However, the presence of microfilariae was not significantly associated with CATT positivity but no definitive conclusion could be drawn.

The main objective of the present study was to provide additional information on the possible false positivity of the CATT using another cloned VAT, LiTat 1.3 derived from T. b. gambiense antigen repertoire LiTAR 1, in the presence of microfilarial infection. This choice resulted from comparative studies on the distribution of several IsoVATs $[4,5]$, in T. b. gambiense repertoires of different geographical origin. We assessed whether the presence of microfilarial infection influenced the CATT results by using i) whole blood and ii) an appropriate casecontrol design: we compared the proportion of subjects with $M$. perstans and L. loa microfilariae $(\mathrm{mf})$ between CATT positive and CATT negative controls matched on age, sex and community of residence. 
Citation: Pion SD, Njiokou F, Boussinesq M, Simo G, Truc P (2017) Confirmation of No Influence of Loa loa and Mansonella perstans on the Card Agglutination Test for Trypanosomosis used for Serological Screening of Human African Trypanosomosis. J Trop Dis 5: 242. doi: 10.4172/2329-891X.1000242

Page 2 of 4

\section{Materials and Methods}

\section{Study sites}

The study was conducted in 2004 in four historical HAT foci: the "Couloir" focus in the Republic of Congo (main locality: Ngabé, located on the bank of the Congo River, about $180 \mathrm{~km}$ north of Brazzaville) and three HAT foci in Cameroon: Fontem (Lebialem Division in the South-West Region), Bipindi and Campo (both located in the Ocean Division, in the South Region).

According to the classification developed to define risk for HAT [6], the Couloir (Mpouya-Ngabé) focus was a very high-risk area (number of cases per inhabitant per year $\geq 1 / 100$ ) whereas the Bipindi and Campo foci were in the moderate risk category (between 1/10,000 and $1 / 1000$ ), and Fontem was a low-risk area (between 1/100,000 and $1 / 10,000)$. The initial surveys to identify CATT positives and CATT negatives subjects were conducted in April and May 2004 in the Campo (nine villages: Afan Essokie II, Bouandjo, Campo-town, Campo-beach, Ebodje, Ipono, Mabiogo, Malaba and Mvasse) and the Bipindi foci (seven villages: Bijouka-Centre, Bipindi-Centre, Ebimimbang, Lambi, Mimfombo, Nyamenkoun and Tyango). In the Ngabé focus, the survey was performed between May and July 2004 and included seven villages (Boka Lefini, Boka Serieux, Bokaba, Brick 1, Brick 2, Brick 3, Mouala). Lastly, the Fontem focus was surveyed in July 2004 (eight villages: Azi, Belleh, Fossung, Menji, Mveh, Nchembin, Nchenfen and Nsoko). Detailed maps of the Bipindi, Campo and Fontem foci have been presented elsewhere [7-9].

\section{Screening strategy and biological examination}

All volunteering residents present in the selected villages were examined during daytime. The first step consisted in a careful palpation of cervical lymph node to detect a possible enlargement. A puncture was done whenever a lymph node was larger than $1 \mathrm{~cm}$ and the fluid was rapidly examined under light microscopy. All individuals with trypanosomes in the fluid were discarded from the present study and were referred to hospital for treatment where a full clinical examination was done for all patients (data not available). After palpation, a $60 \mu \mathrm{L}$ fingerprick blood sample was collected from each individual in a heparinized capillary tube to perform immediately a whole blood CATT (WB-CATT). When the WB-CATT was found positive, an additional $5 \mathrm{~mL}$ blood sample was collected by venous puncture in a heparinized vacutainer tube. Three hundred $\mu \mathrm{L}$ of this sample were processed using the mini-anion-exchange centrifugation technique (mAECT) to detect trypanosomes [10]. The remaining of the blood sample was centrifuged and the plasma obtained was used to perform CATT titration using twofold dilutions from 1 to $1 / 32$ (CATT-P). Dilutions were made in microtiter plates and the operating procedures were performed. All these tests were performed the same day in the field. People in whom trypanosomes were found by the mAECT were also referred to hospital for treatment. All individuals found negative at the mAECT were left untreated.

Filarial infections were searched in $75 \mu \mathrm{L}$ thick blood smears. After dehemoglobinization, the blood smears were stained with Giemsa stain, examined under a light microscope. All L. loa and M. perstans $\mathrm{mf}$ were counted except in the Fontem focus, where only presence/ absence of each species was reported. Mf counts are expressed per 75 $\mu \mathrm{L}$.

\section{Matching procedure and statistical analysis}

During the screening phase, 422 persons were WB-CATT positive among 9,617 persons tested (36/3038 including 2 confirmed HAT cases in Bipindi, 61/2647 including 8 confirmed HAT cases in Campo, 204/2504 with no confirmed HAT case in Fontem and 121/1428 including 77 confirmed HAT cases in Ngabe), but only 146 individuals with a WB-CATT positive result were consenting and included in the present study.

Each of the 146 WB-CATT positive individuals was matched on sex, age ( \pm 2 years) and village of residence with a single WB-CATT negative control (randomly chosen when several non-cases were available for matching). A matched case-control analysis was performed to assess whether the presence and intensity of microfilaremia were associated (a) with WB-CATT positivity and (b) CATT-P end dilution titres.

Chi-square tests were used to compare proportions and Wilcoxon signed-rank tests were used to compare $\mathrm{mf}$ densities between WBCATT positive and WB-CATT negative subjects. In addition, we investigated the relationship between CATT-P intensity (end dilution titre) and presence and intensity of microfilaremia amongst WB-CATT positive individuals only. For this step, we performed a logistic regression analysis with the CATT-P titre (CATT-P $\geq 1 / 16$ vs. CATT-P $<1 / 16)$ as the variable of interest and sex, age and microfilarial status (presence of L. loa and M. perstans $\mathrm{mf}$, and then L. loa $\mathrm{mf}$ count and $M$. perstans $\mathrm{mf}$ count), as covariates.

\section{Results and Discussion}

During the screening, only 146 individuals of 335 with a positive WB-CATT and no HAT confirmation consented to be included in the study. The original design of the study included a follow-up of CATT titration every 3 months during a 1 -year period. The main reason for not consenting was that most individuals with a positive CATT but no parasitological confirmation did not feel ill and refused to be resampled every 3 months. The proportion of individuals showing $L$. loa microfilaremia ranged from $3.6 \%$ in Fontem to $48.3 \%$ in Bipindi. The values for $M$. perstans microfilaremia ranged from $0 \%$ in Fontem to $65.5 \%$ in Bipindi (Table 1). The proportions of $\mathrm{mf}$ carriers were slightly higher in WB-CATT positive individuals than in WB-CATT negative ones for both L. loa (16.4\% vs. $13.2 \%)$ and M. perstans $(14.4 \%$ vs. $12.5 \%)$ but the differences were not statistically significant $(\mathrm{P}=0.22$ and $\mathrm{P}=0.32$, respectively).

The WB-CATT positive individuals tended to have higher microfilarial counts than WB-CATT negative individuals (Table 2): 39.8 vs. 17.3 for L. loa, 21.2 vs. 7.2 for M. perstans (arithmetic means $\mathrm{mf} / 75 \mu \mathrm{l})$. But these differences were not statistically significant $(\mathrm{P}=0.31$ and $\mathrm{P}=0.49$, respectively).

The distribution of CATT-P results according to the end dilution titres (Table 3) was not significantly different between those who were microfilaremic or amicrofilaremic for L. loa $(\mathrm{P}=0.196)$. However, CATT-P $\geq 1 / 16$ results tended to be more frequent in $L$. loa microfilaremic subjects (29.2\%) than in L. loa amicrofilaremic individuals $(14.8 \%, \mathrm{P}=0.087)$. 
Citation: Pion SD, Njiokou F, Boussinesq M, Simo G, Truc P (2017) Confirmation of No Influence of Loa loa and Mansonella perstans on the Card Agglutination Test for Trypanosomosis used for Serological Screening of Human African Trypanosomosis. J Trop Dis 5: 242. doi: 10.4172/2329-891X.1000242

Page 3 of 4

\begin{tabular}{|c|c|c|c|c|c|c|}
\hline \multirow[t]{2}{*}{ Focus } & \multicolumn{3}{|c|}{ No. (\%) of $L$. loa mf carriers } & \multicolumn{3}{|c|}{ No. (\%) of $M$. perstans carriers } \\
\hline & CATT Negative & CATT Positive & Total & CATT Negative & CATT Positive & Total \\
\hline Bipindi & $5 / 14^{a}(35.7 \%)$ & $9 / 15(60.0 \%)$ & $14 / 29(48.3 \%)$ & 11/14a (78.6\%) & $8 / 15(53.3 \%)$ & $19 / 29(65.5 \%)$ \\
\hline Campo & $10 / 21^{\mathrm{b}}(47.6 \%)$ & $10 / 22^{\mathrm{c}}(45.5 \%)$ & $20 / 43(46.5 \%)$ & $6 / 21^{b}(28.6 \%)$ & $12 / 22^{\mathrm{c}}(55.5 \%)$ & $18 / 43(41.9 \%)$ \\
\hline Fontem & $2 / 83^{\mathrm{d}}(2.4 \%)$ & $3 / 83^{\mathrm{e}}(3.6 \%)$ & 6/166 (3.6\%) & $0 / 83(0 \%)$ & $0 / 83(0 \%)$ & $0 / 166(0 \%)$ \\
\hline Ngabe & $2 / 26(7.7 \%)$ & $2 / 26(7.7 \%)$ & $4 / 52(7.7 \%)$ & $1 / 26(3.9 \%)$ & $1 / 26(3.8 \%)$ & 2/52 (3.9\%) \\
\hline Total & 19/144 (13.2\%) & $24 / 146(16.4 \%)$ & $43 / 290(14.8 \%)$ & $18 / 144(12.5 \%)$ & $21 / 146(14.4 \%)$ & $39 / 290(13.5 \%)$ \\
\hline \multicolumn{7}{|c|}{$\begin{array}{l}\text { a } 1 \text { thick blood smear unreadable } \\
\text { b } 1 \text { thick blood smear unreadable; } 3 \text { thick blood smears } L \text {. loa positive without density for } L \text {. loa but with density for } M \text {. perstans } \\
\text { c } 2 \text { thick blood smears } L \text {. Loa positive without density for } L \text {. loa but with density for } M \text {. perstans } \\
\text { d } 3 \text { thick blood smears } L \text {. loa positive without density for } L \text {. loa } \\
\text { e } 3 \text { thick blood smears } L \text {. loa positive without density for } L \text {. loa }\end{array}$} \\
\hline
\end{tabular}

Table 1: Presence of microfilaremia in the different HAT foci according to the CATT status.

Individuals with CATT-P $\geq 1 / 16$ result harboured higher $L$. loa microfilaremia $(58.9 \mathrm{mf} / 75 \mu \mathrm{l})$ than those with a CATT-P result $<1 / 16$ $(23.1 \mathrm{mf} / 75 \mu \mathrm{l})$ but this difference was not significant $(\mathrm{P}=0.16) . M$. perstans microfilaremia did not differ significantly in those with a CATT-P result $<1 / 16(16.1 \mathrm{mf} / 75 \mu \mathrm{l})$ and those with a CATT-P result $\geq$ $1 / 16(6.1 \mathrm{mf} / 75 \mu \mathrm{l}, \mathrm{P}=0.39)$.

\begin{tabular}{|l|l|l|l|l|}
\hline Location & \multicolumn{2}{|l|}{ L. loa $\mathrm{mf} / 75 \mu \mathrm{L}$ of blood } & \multicolumn{2}{l|}{$M$. perstans $\mathrm{mf} / 75 \mu \mathrm{L}$ of blood } \\
\hline & CATT Negative & CATT Positive & CATT Negative & CATT Positive \\
\hline Bipindi & $58.2(121.5)$ & $108.7(219.0)$ & $21.6(26.7)$ & $63.9(122.4)$ \\
\hline Campo & $9.9(26.4)$ & $35.9(107.2)$ & $6.2(17.9)$ & $16.5(27)$ \\
\hline Fontem & NA & NA & NA & NA \\
\hline Ngabe & $0.3(1.12)$ & $3.1(14.9)$ & $0.2(1.0)$ & $0.2(1.0)$ \\
\hline Total & $17.3(64.3)$ & $39.8(129.2)$ & $7.2(18.2)$ & $21.2(66.2)$ \\
\hline
\end{tabular}

Table 2: Intensities of microfilaremia (mean ( \pm standard deviation)) in each HAT focus according to the CATT status.

Adjusting on individual factors, logistic regressions taking into account presence/absence or number of $L$. loa and M. perstans $\mathrm{mf}$ did not reveal any significant association between L. loa or M. perstans infection and a CATT-P result $\geq 1 / 16$. Thanks to sustained control activities, HAT prevalence appears now very low in most of African countries. A rapid diagnostic test (RDT) [11], is now recommended for passive HAT detection.

However, active medical surveys are ongoing in the Republic of Congo, Central African Republic and Chad, still based on CATT using the LiTat 1.3 antigen. In addition, in case of RDT positive confirmed by parasitology (HAT patient), a small medical survey will be performed in the village of this latter patient using CATT. CATT remains the only serological test for HAT mass screening, using the best reagent based on LiTat 1.3 and not AnTat 1.8 to prevent cross-reaction with a warrant of good specificity.

\begin{tabular}{|c|c|c|c|c|c|c|c|c|}
\hline \multicolumn{9}{|c|}{ CATT-P end titre results } \\
\hline & & 1 & $1 / 2$ & $1 / 4$ & $1 / 8$ & $1 / 16$ & $1 / 32$ & Total \\
\hline \multirow[t]{2}{*}{ Bipindi } & $\begin{array}{l}\text { Loa } \\
\text { mf - }\end{array}$ & 0 & 1 & 2 & 1 & 0 & 2 & 6 \\
\hline & $\begin{array}{l}\text { Loa } \\
\mathrm{mf}+\end{array}$ & 0 & 3 & 1 & 2 & 0 & 3 & 9 \\
\hline \multirow[t]{2}{*}{ Campo } & $\begin{array}{l}\text { Loa } \\
\text { mf - }\end{array}$ & 0 & 4 & 2 & 3 & 1 & 2 & 13 \\
\hline & $\begin{array}{l}\text { Loa } \\
\mathrm{mf}+\end{array}$ & 0 & 2 & 1 & 4 & 2 & 1 & 9 \\
\hline \multirow[t]{2}{*}{ Fontem } & $\begin{array}{l}\text { Loa } \\
\text { mf - }\end{array}$ & 8 & 26 & 27 & 15 & 2 & 2 & 80 \\
\hline & $\begin{array}{l}\text { Loa } \\
\mathrm{mf}+\end{array}$ & 0 & 1 & 2 & 0 & 0 & 0 & 3 \\
\hline \multirow[t]{2}{*}{ Ngabe } & $\begin{array}{l}\text { Loa } \\
\text { mf - }\end{array}$ & 3 & 2 & 7 & 3 & 5 & 4 & 24 \\
\hline & $\begin{array}{l}\text { Loa } \\
\mathrm{mf}+\end{array}$ & 0 & 0 & 0 & 1 & 1 & 0 & 2 \\
\hline \multirow[t]{2}{*}{ All foci } & $\begin{array}{l}\text { Loa } \\
\text { mf - }\end{array}$ & 11 & 33 & 38 & 22 & 8 & 10 & 122 \\
\hline & $\begin{array}{l}\text { Loa } \\
\mathrm{mf}+\end{array}$ & 0 & 6 & 4 & 7 & 3 & 4 & 24 \\
\hline
\end{tabular}

Table 3: Distribution of CATT-P end titre results in subjects with and without Loa loa microfilaremia.

The CATT using blood showed a specificity of $96.5 \%$, which is consistent with the usually reported values. We compared CATT positive individual with negative ones, and evaluate the influence of $L$. loa and $M$. perstans on CATT using whole blood and serum dilution. No significant difference was observed in each comparison, confirming previous results despite the different antigens used in the CATT reagent as mentioned above. 
Citation: Pion SD, Njiokou F, Boussinesq M, Simo G, Truc P (2017) Confirmation of No Influence of Loa loa and Mansonella perstans on the Card Agglutination Test for Trypanosomosis used for Serological Screening of Human African Trypanosomosis. J Trop Dis 5: 242. doi: $10.4172 / 2329-891 X .1000242$

Page 4 of 4

\section{Conclusion}

WB-CATT positive persons were not more frequently infected by $L$ loa and $M$. perstans $\mathrm{mf}$ than WB-CATT negative ones. The distribution of CATT-P dilution titres was not significantly different between those who were microfilaremic or amicrofilaremic. This unique matched case-control study does not bring any evidence of the influence of $L$. loa or $M$. perstans on serodiagnosis of HAT in the field using CATT/T. b. gambiense LiTat 1.3. Therefore, HAT screening activities can be performed without controlling for filariasis at the same time.

\section{Acknowledgements}

We deeply thank all the population of the villages and the cohort subjects, the local Authorities, the Ministry of Health of Cameroon and Republic of Congo and their respective National Control Program against HAT (Heads: Mr V. Eboo Eyenga, Dr A. Mantelot respectively). We thank the Organization for the Coordination of Control of Endemic Diseases in Central Africa OCEAC, and Dr Nicolas M'Bongo from the National Public Health Laboratory of the Republic of Congo. (TDR) A30176. Tropical Diseases (TDR).

\section{References}

1. Magnus E, Vervoort T, Van MN (1978) A card-agglutination test with stained trypanosomes (C.A.T.T.) for the serological diagnosis of T.b.gambiense trypanosomiasis. Ann Soc Belge Med Trop 58: 169-176.

2. Control and surveillance of human African trypanosomiasis (2013) Report of a WHO expert committee. World Health Organization, Geneva.
3. Noireau F, Lemesre JL, Nzoukoudi MY, Louembet MT, Gouteux JP, et al. (1988) Serodiagnosis of sleeping sickness in the Republic of the Congo: Comparison of indirect immunofluorescent antibody test and card agglutination test. Trans R Soc Trop Med Hyg 82: 237-240.

4. Magnus E, Vervoort T (1982) Serological cross-reactivities among Trypanosome Variable Antigen Isotypes of the subgenus Trypanozoon. Ann Soc Belge Med Trop 62: 25-29.

5. Van MN, Magnus E, Vervoort T (1977) Comparisons of variable antigen types produced by trypanosme strains of the subgenus trypanozoon. Ann Soc Belge Méd Trop 57: 409-423.

6. Simarro PP, Cecchi G, Franco JR, Paone M, Fevre EM, et al. (2011) Risk for human african trypanosomiasis, central Africa. Emerg Infect Dis 17: 2322-2324.

7. Simo G, Asonganyi T, Nkinin SW, Njiokou F, Herder S, et al. (2006) High prevalence of Trypanosoma brucei gambiense group 1 in pigs from the Fontem sleeping sickness focus in Cameroon. Vet Parasitol 139: 57-66.

8. Grébaut P, Melachio T, Nyangmang S, Eyenga VE, Njitchouang GR, et al. (2016) Xenomonitoring of sleeping sickness transmission in Campo (Cameroon). Parasit Vectors 9: 201.

9. Grébaut P, Bodo JM, Assona A, Foumane NV, Njiokou F, et al. (2001) Research of risk factors for African human trypanosomiasis in the Bipindi outbreak in Cameroon. Med Trop 61: 377-383.

10. Lumsden WH, Kimber CD, Evans DA, Doig SJ (1979) Trypanosoma brucei: Miniature anion-exchange centrifugation technique for detection of low parasitaemias: Adaptation for field use. Trans R Soc Trop Med Hyg 73: 312-317.

11. Büscher P, Gilleman Q, Lejon V (2013) Novel rapid diagnostic test for sleeping sickness. N Engl J Med 368: 1069-1070. 